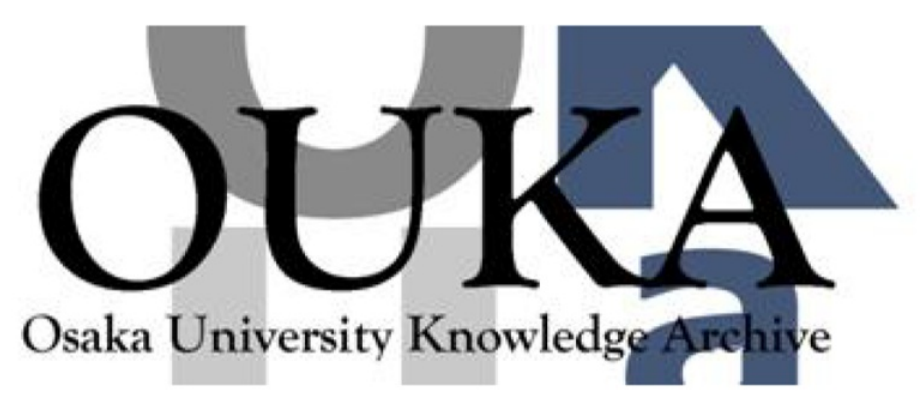

\begin{tabular}{|c|l|}
\hline Title & $\begin{array}{l}\text { Resolution improving method from multi-focal } \\
\text { omnidirectional images }\end{array}$ \\
\hline Author(s) & 長原, 一 八木, 康史; 谷内田, 正彦 \\
\hline Citation & $\begin{array}{l}\text { IEEE International Conference on Image } \\
\text { Processing. } 1 \text { p. } 654-p .657\end{array}$ \\
\hline Issue Date & $2001-10$ \\
\hline oaire:version & VoR \\
\hline URL & $\begin{array}{l}\text { https://hdl. handle. net/11094/14101 } \\
\text { c2001 IEEE. Personal use of this material is } \\
\text { permitted. However, permission to } \\
\text { reprint/republish this material for advert ising } \\
\text { or promotional purposes or for creat ing new } \\
\text { collective works for resale or redistribution } \\
\text { to servers or lists, or to reuse any } \\
\text { copyrighted component of this work in other } \\
\text { works must be obtained from the IEEE. . }\end{array}$ \\
\hline rights \\
\hline Note & \begin{tabular}{l} 
\\
\hline
\end{tabular} \\
\hline
\end{tabular}

Osaka University Knowledge Archive : OUKA

https://ir. Library. osaka-u. ac. jp/

Osaka University 


\title{
RESOLU'TION IMPROVING METHOD FROM MULTI-FOCAL OMNIDIRECTIONAL IMAGES
}

\author{
Hajime Nagahara, Yasushi Yagi, and Masahiko Yachida \\ Graduate School of Engineering Science, Osaka University \\ 1-3 Machikaneyama-cho, Toyonaka city, Osaka, 560-8531, JAPAN
}

\begin{abstract}
The omnidirectional image sensor named HyperOmniVision, is composed of hyperboloidal mirror and conventional video camera. It can observe 360 degree field of view and can transform an input image to a perspective image. However, it has an intrinsic problem where the image resolution of HyperOmniVision is lower than that of an ordinary video camera, because of its structure that only one CCD captures whole periphery scene. In this paper, we propose the resolution improvement method by using sub-pixel displaced and multi-focused images.
\end{abstract}

\section{INTRODUCTION}

Many image-based applications require that a large field of view is imaged. Examples include surveillance, tele-conference and telepresence in virtual reality and robotics. Unfortunately, conventional video camera has limited field of view, which make them restrictive for these applications. One solution to enlarge the field of view is to use an omnidirectional image sensor.

The omnidirectional image sensor, HyperOmniVision[1], can observe 360 degree field of view horizontally at video rate. In addition, HyperOmniVision is composed of hyperboloidal mirror and conventional video camera, and have a single effective viewpoint (center of projection). Therefore, an input image captured by HyperOmniVision can be transformed to geometrically correct and seamless perspective image. These characteristics are suitable for the applications that are needed to present to human immersively. However, the angular resolution of HyperOmniVision is lower than that of conventional video camera, because of its structure that whole omnidirectional scene is captured by only one CCD.

Since Tsai and Huang [2], many super-resolution methods combining multiple low-resolution images have been proposed for improving the resolution. Most super-resolution algorithms are based on the constraints that the super-resolution image, when appropriately warped and down-sampled to model the image formation process, should yield the low resolution input images[3][4]. Schultz and Steverson[5] have been embedded it in a Bayesian framework. Cheeseman et al. [6] have been proposed including simultaneously computing structure.

We have been applied such super-resolution methods to $\mathrm{Hy}$ perOmniVision in consideration of the difference of its imaging process[7]. In this result, the resolution of HyperOmniVision is increased by applying super-resolution from an omnidirectional image sequence. However, the input images have a defocus caused by aberrations of HyperOmniVision. The resolution improvement was restricted by such optical blur. In this paper, we propose the resolution improving method with using not only sub-pixel displaced but also multi-focused image sequence to overcome this blur limitation.

\section{CHARACTERISTICS OF HYPEROMNIVISION}

HyperOmniVision consists of a standard video camera combined with a convex hyperboloidal mirror as shown in figure 1. Hyperboloidal mirror is defined by equations (1),(2). The mirror has two focal points. The optical center of the camera lens aligns with one of the focal points $-c$. It is enable to get the omnidirectional image at video rate by capturing upward the scene reflected on the mirror. Figure 2-a shows the example of input image. A depression angle $\alpha$ and a direction $\beta$ of incident ray are uniquely derived from an arbitrary point $p(x, y)$ on input image by the property of hyperboloid in equations (3)-(5). From these characteristics, HyperOmniVision can observe 360 degree field of view at video rate and can easily transform its input image to a common perspective(Fig 2-b) and a panoramic output image(Fig 2-c).

However, the angular resolution of HyperOmniVision is lower than that of conventional video camera, because of its structure that whole periphery scene is captured by only one CCD shown in figure1. Moreover, the resolution is decreased with increasing the angle of depression angle of viewpoint. Solid lines in figure 3 shows the resolution against depression angle on circumferential and radial directions respectively. It is remarkable that the resolution is lower in the bottom area of transformed image.

In this paper, we aim to develop the method for improving not only the resolution of whole image but also the resolution on the transformed images.

$$
\begin{aligned}
& \frac{X^{2}+Y^{2}}{a^{2}}-\frac{Z^{2}}{b^{2}}=-1(Z>0) \\
& c=\sqrt{a^{2}+b^{2}} \\
& \beta=\tan ^{-1}\left(\frac{y}{x}\right) \\
& \alpha=\tan ^{-1} \frac{\left(b^{2}+c^{2}\right) \sin \gamma-2 b c}{\left(b^{2}-c^{2}\right) \cos \gamma} \\
& \gamma=\tan ^{-1} \frac{f}{\sqrt{x^{2}+y^{2}}}
\end{aligned}
$$

\section{PREVIOUS PROBLEM OF RESOLUTION IMPROVEMENT FOR HYPEROMNIVISION}

Previously, we applied super-resolution methods to HyperOmniVision to resolve the low-resolution problem[7]. In this paper, we 


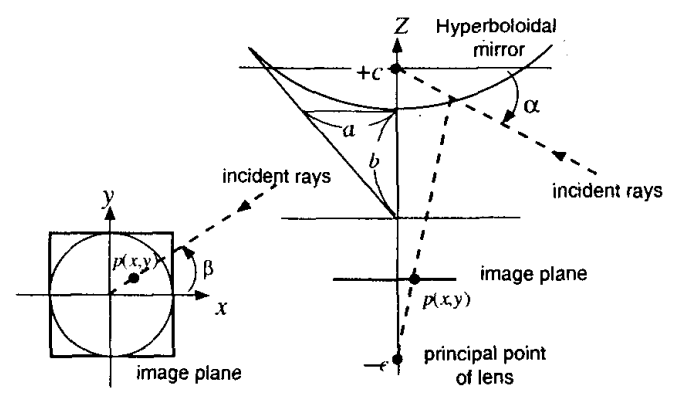

a:Top view

b:Side view

Fig. 1. Optics of HyperOmniVision

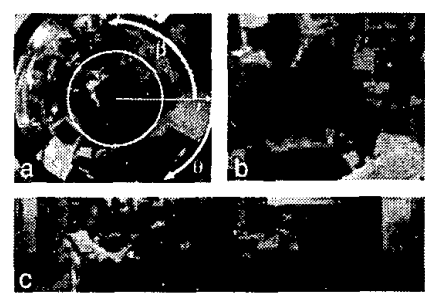

Fig. 2. An example of omnidirectional image and transformed images. a: An input image, b: A transformed perspective image, c: A transformed panoramic image.

modeled imaging process of HyperOmniVision, such as optics, $\mathrm{CCD}$ and visual signal transformation from an input to output images, then optimized an optical design and an image processing for super-resolution. We applied two high-resolution algorithms such as nearest neighbor interpolation by using multiple images and back projection method. We compared with standard bilinear interpolation, the resolution of the transformed image was improved. However, the size of the blur in the image depends on depression angle described by dotted lines in figure 3 . The blur is caused from the aberration of HyperOmniVision. This blur restricts the resolution improvement. Of course, back projection method is able to include the blur restoration process, but it is not effective when the blur is large like HyperOmniVision in spite of its high computation cost.

The CCD frequency response using sub-pixel displacement is shown in figure 4. From figure 4, cut off frequency is approx-

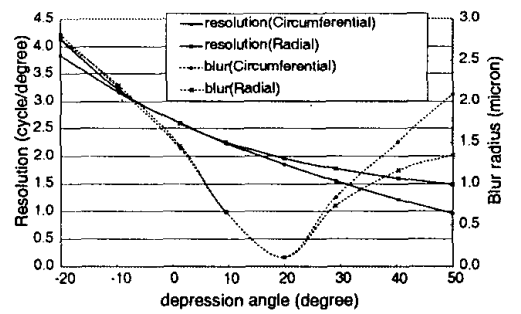

Fig. 3. Resolution and blur radius versus depression angle
Table 1. Mirror and lens parameters

\begin{tabular}{|c|c|c|}
\hline $\begin{array}{c}\text { Hyperboloidal } \\
\text { mirror }\end{array}$ & $\mathrm{a}$ & $63.8 \mathrm{~mm}$ \\
\cline { 2 - 3 } & $\mathrm{b}$ & $88.4 \mathrm{~mm}$ \\
\hline \multirow{3}{*}{$\begin{array}{c}\text { CCD } \\
\text { camera }\end{array}$} & Lens & paraxial \\
\cline { 2 - 3 } & $\mathrm{F}$ & 1.4 \\
\cline { 2 - 3 } & CCD cell pitch & 15 micron \\
\cline { 2 - 3 } & CCD cell size & 7.5 micron \\
\hline
\end{tabular}

imately 2.5 times improved against Niquist limit by using subpixel displaced images. Figure 5 shows the cut of frequency of CCD with sub-pixel displacement and MTF(Modulated Transfer Function) response against depression angle when the focal point of the lens is set on the point where depression angle is 20 degree. The MTF peak is appeared at 20 degree. CCD frequency response is only higher than that of MTF in the 5 to 40 degree range. It means that the optical property is not satisfied with CCD frequency requirement by sub-pixel displacement at any depression angle. Figure 5 also shows that MTF response in the case that the blur is double. From this plot, if the blur is larger, the satisfiable area with CCD is getting narrower. These plots indicate that the intrinsical blur restricts the resolution improvement. From above description, we know that clear image can be obtained at a certain interval of depression angle about the focused point.

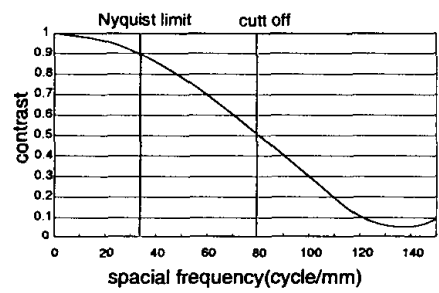

Fig. 4. Frequency response of $\mathrm{CCD}$

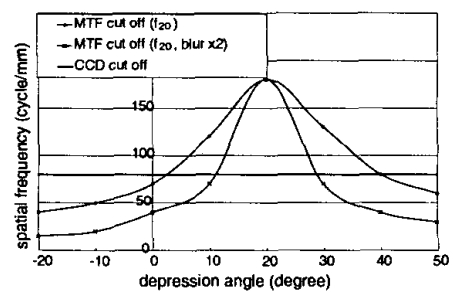

Fig. 5. Cut off frequency of MTF and CCD versus depression angle on circumferential direction

\section{RESOLUTION IMPROVEMENT BY USING MULTI-FOCUSED IMAGES}

The proposed method improves the transformed image resolution by combining sub-pixel displaced and multi-focused images. Discrete sampling points are varied with the camera rotation. It is assumed that the nearest input pixel across the output image pixel has the most accurate information through the consecutive images. 


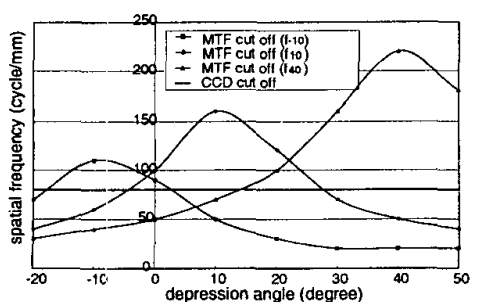

Fig. 6. Cut off frequency of MTF and CCD versus depression angle on circumferential direction

Therefore; the high-resolution image is constructed by selecting nearest neighbor pixel from displaced input consecutive images directly. Moreover, the previous blur problem is solved by also selecting focused planes into the multi-focused images depend on the depression angle. Thus idea is illustrated in figure 8 . As shown in figure 7, we note that the focal length is different corresponding to the depression angle owing to the inherent aberration of HyperOmniVision. In this paper, we denote $f_{-10}$ as the focal length when the focus is aligned with the point where the depression angle is -10 degree. Figure 6 shows the CCD cut off and MTF cut off frequency against depression angle in the cases that the focal length of the lens is set on $f_{-10}, f_{10}$ and $f_{40}$ respectively. These plots are calculated by simulation of HyperOmniVision described in table 1. As shown in figure 6, MTF frequency response is changed corresponding to focal point of the lens. The MTF frequency response are satisfied with $\mathrm{CCD}$ frequency requirement on the region near the focusing point, such as the area of depression angle from -20 to 2 degree in the case of $f_{-10}$, and from -2 to 28 degree in the case of $f_{10}$. Therefore we select the focuses so that MTF responses are satisfied with $\mathrm{CCD}$ frequency requirement through the full area.

Next, we consider how to combine the number of different focal images. Figure 9 shows input/output pixel ratio against the number of input images on each regions with matching focus of $f_{-10}, f_{10}$ and $f_{40}$. The number of input images to fill output pixels depends on these regions, because the coordinates are different between an input and output transformed images. Figure 9 shows that output pixels are $90 \%$ filled by only 4 input images on the region with matching focus by $f_{-10}$ (depression angle from -20 to 2 degree region). On the other hands, about 50 images are required on the region by $f_{40}$ (depression angle from 21 to 50 degree region). Therefore, we select the minima number of combination of multi-focused images. For example, if we would use 25 input images in the case shown in figure 9 , we select the combination that 2 input images focused on $f_{-10}, 5$ images focused on $f_{10}$ and 18 images focused on $f_{40}$.

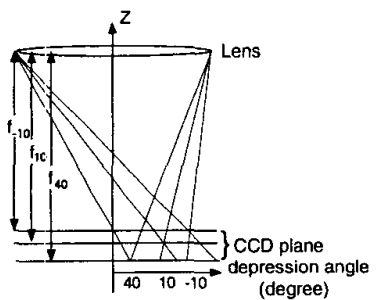

Fig. 7. The difference of focal length

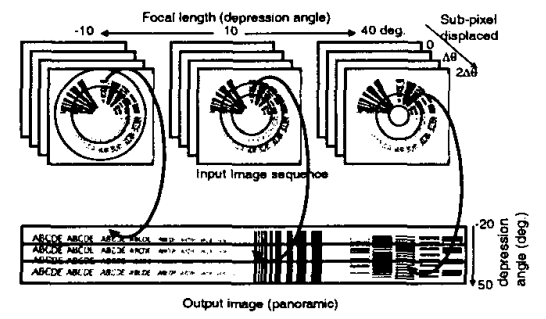

Fig. 8. Super-resolution process with sub-pixel displacement and multi-focused image sequence

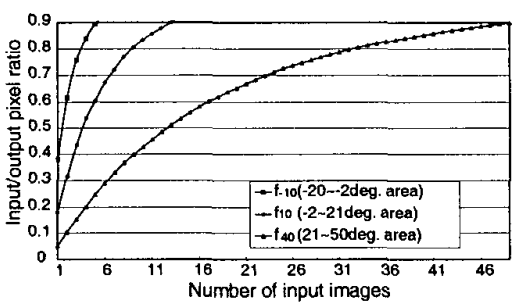

Fig. 9. Input/output pixel ratio

\section{EXPRIMENTS}

\subsection{Simulation}

The simulated input images are calculated by simulation of imaging process on HyperOmniVision indicated in table 1, then we estimated the resolution improving method suggested here numerically and graphically. We used a panoramic image as transformed image in this simulation experiments. Figure 10 shows samples of simulated input images when the focal lengths are set on $f_{-10}$ and $f_{40}$. Note that the areas without focus are blurred by aberration effect. Figure 11 shows PSNR(Peak Signal Noise Ratio) of each method against the number of used input images to estimate improvement of resolution numerically. PSNR indicates similarity between the result and ideal high-resolution images. $f_{20}$ was best focal length for single focus case. In addition. Figure 11 also shows the case that a size of blur was double. From these plots, PSNR were increased proportional to the number of input images and approximately saturated at 25 input images. The PSNR result of proposed method was approximately $1.0 \mathrm{~dB}$ higher than that of single focused method(solid lines in figure 11). It means that the resolution was improved by using multi-focused images. Furthermore, the PSNR of single focused case decreased $2.3 \mathrm{~dB}$ when the size of blur was double(dotted lines in figure 11). On the other hand, the decrease in PSNR of the proposed method was only 0.6 $\mathrm{dB}$. Figure 12 shows result of panoramic images transformed from the region drawn by a fan-shaped dotted region in figure 10. Figure 12-left, center and right are enhanced images by a standard bilinear interpolation, a single focus approach with 25 sub-pixel displaced input images and the proposed method respectively. From these results, the resolution improvement using multi-focused input images was confirmed numerically and graphically on the simulation. 

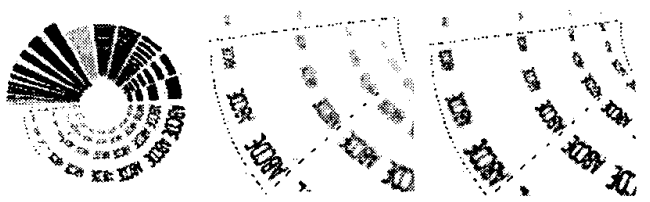

Fig. 10. Example of multi-focal input images(11010 pixels(dotted area)). Left: Whole image, center: Zoom up image $\left(f_{-10}\right)$, right: Zoom up image $\left(f_{40}\right)$.

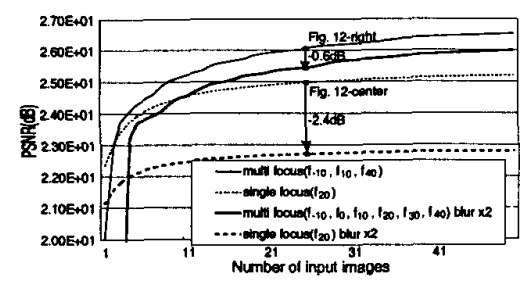

Fig. 11. PSNR of each method

\subsection{Experiment on real scene}

In practice, we constructed the imaging system and an experiment was carried out in real environment. The system consists of hyperboloidal mirror (the mirror parameter indicate in table 1), video camera (XP-009/Sony) and turning table (DR-160/SIGMA KOKI) shown in figure 13. Real input images are captured with rotation of the sensor system to make the sub-pixel displaced. The sensor rotation angle was chosen 0.13 (degree/frame). Figure 14 shows a real input image captured by this system. Figure 15 shows results of perspective image transformed from the region drawn by a fanshaped dotted region in figure 14. Figure 15-left, center and right are results of standard bilinear interpolation, single focus approach with sub-pixel displaced 25 input images and the proposed method respectively. The proposed method can enhance the resolution of output.

\section{CONCLUSION}

In this paper, we suggested the resolution improvement method for omnidirectional image sensor HyperOmniVision, by using both sub-pixel displaced and multi-focused input images. From the experiments on simulated image and real image, we confirmed that

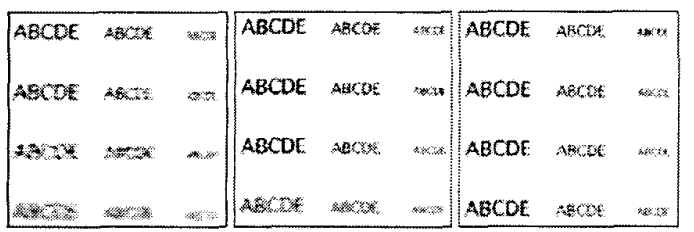

Fig. 12. simulation image result(panoramic image, $256 \times 256$ pixels). Left: Bilinear interpolation( 1 input image, focal length $f_{20}$, PSNR 21.2dB), center: Nearest neighbor interpolation from subpixel displaced images(25 input images, focal length $f_{20}$, PSNR $25.0 \mathrm{~dB}$ ), right: Nearest neighbor interpolation from multi-focused and sub-pixel displaced images (25 input images, focal length $f_{-10}, f_{10}, f_{40}$, PSNR 26.0dB).

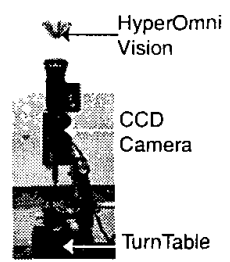

Fig. 13. Imaging system

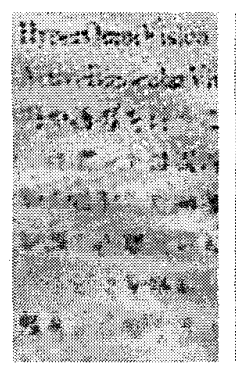

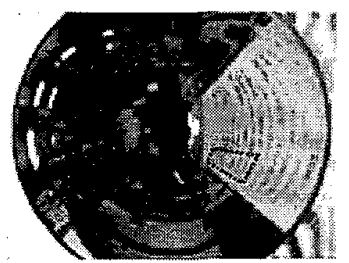

Fig. 14. A sample of real input image(4108 pixels(dotted area))

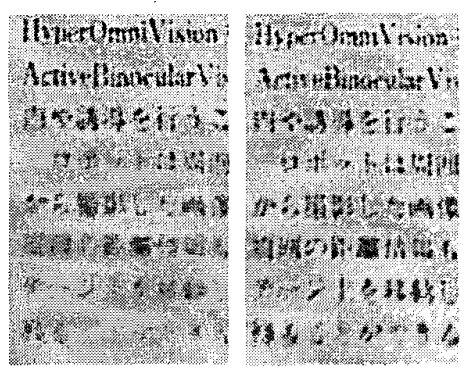

Fig. 15. real image result (perspective image, $116 \times 185$ pixels). Left: Bilinear interpolation, center: Nearest neighbor interpolation from sub-pixel shifted images, right: Nearest neighbor interpolation from multi-focused and sub-pixel sifted images.

the resolution was improved by using multi-focused images.

\section{REFERENCES}

[1] Y. Yamazawa, Y. Yagi, and M. Yachida, "New real-time omnidirectional image sensor with hyperboloidal mirror," in Proc. 8th Scandinavian Conf. on Image Analysis, 1995, vol. 2, pp. 1381-1387.

[2] R. Y. Tsai and T. S. Hang, "Multiframe image resolution and registration," Advanced in Computer Vision and Image Processing, vol. 1, pp. 317-339, 1984.

[3] S. Pelg, D. Keren, and L. Schweitzer, "Improve image resolution using subpixel motion," Pattern Recognition letters, pp. 233-226, 1987

[4] M. Irani and S. Pelg, "Improving resolution by image registoration," Computer Vision, Graphics, and Image Processing, vol. 52, pp. 231-239, 1991.

[5] R. Schultz and R.Stevenson, "Extruction of high-resolution frames from video sequence," IEEE Trans. Image Processing, vol. 5, no. 6, pp. 996-1011, 1996.

[6] P. Cheeseman, B. Kanefsky, R. Kraft, J. Stutz, and R. Hanson, "Super-resolved surface reconstrucion from multiple images," Tech. Rep., FIA-94-12, NASA Ames Reserch Center, 1994.

[7] Hajime Nagahara, Yasushi Yagi, and Masahiko Yachida, "Super-resolution from an omnidirectional image sequence," in Proc. IEEE Int. Conf. Industrial Electronics, Control and Instrumaentation, 2000, pp. 2559-2564. 